\title{
SANS contrast applied to study hierarchical structure of plant biomass during assembly and deconstruction
}

\author{
Sai Venkatesh Pingali ${ }^{1}$ (pingalis@ornl.gov)*, Barabara Evans, ${ }^{1}$ Loukas Petridis, ${ }^{1}$ Volker Urban, ${ }^{1}$ \\ Jeremy Smith, ${ }^{1}$ Brian H. Davison, ${ }^{1}$ and Hugh O’Neill, ${ }^{1}$ \\ ${ }^{1}$ Oak Ridge National Laboratory, Oak Ridge, Tennessee \\ Email Contact: pingalis@ornl.gov
}

Natural lignocellulosic biomass is a complex self-assembled hierarchical material composed of crystalline cellulose microfibrils laminated with hemicellulose, pectin, and lignin. It is a potential feedstock for the production of bioethanol. However, a limiting factor in cellulosic ethanol production is the recalcitrance of lignin and possibly hemicellulose that hinders hydrolysis of cellulose into sugars for fermentation. Neutron sensitivity for the various polymers are very similar and so three major strategies employed to introduce contrast in this system will be discussed. First, maximum contrast between native plant samples and solvent used for the early in-situ SANS measurements; this approached allowed observation of changes to cellulose microfibril and lignin association process. Second, introduced contrast between cellulose and amorphous polymers- hemicellulose and lignin, by synthesizing composite materials comprising of deuterated bacterial cellulose with hydrogenated amorphous materials. These studies showed differences in cellulose/-xyloglucan and -glucomannan interaction. Third, use partially deuterated switchgrass with differential contrast between cellulose and lignin. Partial deuteration have shown to improve structural resolution during chemical reactions that would not have been possible otherwise. Overall, the improvement in data quality was achieved by either decreasing incoherent background or improve the coherent signal, with both contributing to better signal-to-noise. 